\title{
Scorpion Sting Decreases Pulse Rate and its Possible Management: A Case Report
}

\author{
Khaled Feroz Chenna', Mohammad Jaffar Sadiq ${ }^{2}$, Faisal Imran ${ }^{3}$ \\ ${ }^{1}$ Department of Quality and Development, Batterjee Medical College, North Obhur, Jeddah, KINGDOM OF SAUDI ARABIA. \\ ${ }^{2}$ Associate Professor, Department of Clinical Pharmacology, Batterjee Medical College,, North Obhur, Jeddah, KINGDOM OF SAUDI \\ ARABIA. \\ ${ }^{3}$ Department of Quality and Development, Batterjee Medical College, North Obhur, Jeddah, KINGDOM OF SAUDI ARABIA.
}

\begin{abstract}
Scorpion bites are more common in Saudi Arabia as like in other countries. Normally, the stings are harmless and well tolerated by adult humans, but, sometime around $30 \%$ of untreated cases may show up very serious clinical conditions, including death rarely. Hence, we report a case of scorpion sting that has presented with decreased pulse rate which was manageable easily.
\end{abstract}

Key words:_Scorpion sting, Decreased pulse rate, Scorpion sting management.

Key Message: Whenever a case of scorpion sting gets identified, one must take proper care and confusion must be avoided. Time should not be wasted in waiting and administration of anti toxin for the scorpion sting must be prophesized. Drugs of choice must be the first line drugs like Prazocin should be preference and the subject must be placed under observation because non-treated scorpion sting cases has reported to be lethal in about $30 \%$ of the cases.

\section{INTRODUCTION}

Scorpion species are widely distributed throughout the world summing up to a number of 1500 of which 50 species are considered lethal for humans. ${ }^{1}$ Most lethal ones and the medically important species belongs to the family 'Buthidae. ${ }^{1,2}$ The widely distributed poisonous species of scorpions like 'Leiurus Quinquestriatus' and 'Androctonus Crassicanda' of Saudi Arabia are grouped under the families 'Buthidae' and 'Scorpionidae.'

There have been reported fatalities due to the scorpion sting in the different regions of Saudi Arabia. However, since 1983, there has been decreased in the mortality due to scorpion sting though the morbidity has been the same. ${ }^{3}$ Hence, here we present a case with the clinical manifestation following scorpion sting with decreased pulse rate, identified in the Jeddah region of Saudi Arabia.

\section{CASE HISTORY}

A 36 years old, male subject was present with chief complaints of pain, inflammation and itching around the lower right leg at the emergency clinic of a tertiary care teaching hospital. On examination, followed by the interview he revealed that the subject was stung twice by a pale green colored scorpion on the lateral side of the right leg. The inflammatory points were clearly seen on the legs at two different sights and it was confirmed that the subject was stung twice on the lower extremities. His past medication history was not significant in relation to this case where as, past medical history revealed that the subject was once stung by the scorpion long time ago and till the time he reached the emergency clinic he was not administered with any drug as he was in a state of good health.

The physician on examination observed no change in his vital reports as blood pressure
DOI: 10.5530/ijopp.10.4.60

Address for correspondence: Dr Mohammad Jaffar Sadiq, Department of Clinical Pharmacology, Batterjee Medical College, P.0. 6231, North Obhur, Jeddah - 21442, KINGDOM OF SAUDI ARABIA.

Phone no: 966543942251 Email Id: jaffars909@gmail.com

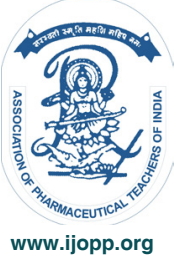


122/90mm Hg, Pulse rate 100 beats / min, respiratory rate $22-24 \mathrm{c} / \mathrm{min}$, blood sugar levels were $110 \mathrm{mg} / \mathrm{dl}$, $\mathrm{SpO}_{2}$ levels were identified to be normal at 99. As most of the signs were normal and lack of scorpion specific anti-venom, the subject was placed under observation. Later, after the interval of $3 \mathrm{~h}$, his tests were repeated again, and all the reports were obtained normal like blood pressure $114 / 90 \mathrm{~mm} \mathrm{Hg}$, respiratory rate $18-20 \mathrm{c} / \mathrm{min}$, blood sugar levels were $108 \mathrm{mg} / \mathrm{dl}, \mathrm{SpO}_{2}$ levels were identified to be normal at 98 except the pulse rate which has dropped down to $62-64$ beats/min but the ECG appeared to be normal throughout the duration of case observation. Again, the tests were run after an interval of $3 \mathrm{~h}$ most of his vital reports were normal but the subject was expressing a certain degree of fatigue and feeling of restlessness but conversating normally.

\section{DISCUSSION}

Scorpion venom is an aqueous soluble, multi protein, antigenic mixture which may affect different vital organs of human body like heart, blood vessels, lungs, and the decrease in the pulse rate which is the only change noticed, indicates that there may have been either due to the toxin mediated autonomic effect on the heart or the parasympathetic derive by the patient himself towards getting the treatment for the scorpion sting. One of this two points may have produce an-add on effect to the diagnosis of decreased pulse rate in the poisonous stung subject. ${ }^{1,2,3,4,5}$

As there were no alteration in the signs like development of fever, shock, convulsions, cyanosis, arrythmias, tachycardia, hypertension or hypotension, tachypnoea, dyspnoea, pulmonary edema or difficulty in breathing, dehydration, dilated pupil, ST segment elevation (ECG) the patient was stabilized soon, but on the other hand any alteration may have demand for an urgent clinical intervention, thank fully it was not in this case. But, other symptoms like pain, swelling, restlessness, bradycardia, were observed for a short period of time and got self stabilized in a quick span of time. The reaction pattern is a self explanatory and may justify the fact that the scorpion may belong to the non-poisonous scorpion family and the reactions may be considered as anaphylactic to the poison with less lethality. Even patient reported to be carrying the killed corps of the scorpion for the ease of identification of the species and specific treatment shall be given followed by the identification but the treatment of delayed because of non-availability of scorpion anti-venom, however at the time of scorpion sting management there was a discussion among the clinical staff that it can also be managed with the application of steroids later the idea was dropped.

\section{Possible management of scorpion sting}

The subject must be received with care and his case history must be noted and the management shall be done with reference to the core drug use guidelines prescribed by the WHO. ${ }^{6}$ Or otherwise the management of the scorpion sting shall be achieved with the infiltration of the local anaesthetic agent without adrenalin and longer comfort can be achieved with the local administration of dehydroemetin $0.03 \%$ as injection. The drug of choice in the management of scorpion sting is Prazocin, at a dose of 0.25 to $0.5 \mathrm{mg}$ (adult dose) orally and repeats the dose for every $6 \mathrm{~h}$ if the improvement is not noticed in the condition of the subject. In case of life threatening pulmonary edema, sodium nitroprusside or aminophylline through intravenous infusion under careful examination shall be given, may be sublingual nifedipine can reduce the $\mathrm{BP}$ and afterload but given rapidly the negative Inotropic effect of nifedipine can precipitate or worsen pulmonary edema. Whereas, the drugs like atropine, antibistamines, glucocorticoids, digoxin, diuretics, dopamine, and dobutamine may worsen the situation of scorpion bite. If at all, these agents are preferred to be used they must be used with lot of care and ultimately relies on the choice and options of the physicians. ${ }^{5}$ Administration of scorpion anti venom is the best step in the management of the scorpion sting cases.

An open label clinical trial explains that the combination of scorpion anti-venom to "prazosin" improves recovery time and lessens the hospital stay in case of scorpion sting in humans. ${ }^{7}$ A systematic review and meta analysis of controlled clinical trials explains that the steroids have no beneficial role in the management of scorpion stings, local application of anesthesia either through trans-dermal patches or through subcutaneous route was identified to beneficial in relieving the local pain in comparison to the administration of other drugs through IV route or applying ice topically over the stung area, Prazosin administration may be beneficial in all the cases including the children, at certain instances poly-valent anti venom were identified to in effective against placebo included control trials. ${ }^{8}$

\section{Points of interest in the current case report}

A review of few references has been presented for the quick of the clinicians which includes,

- Prazosin is best choice of drug in the management of scorpion sting cases

- sodium nitroprusside or aminophylline can also be employed with strict clinical supervision 
- Avoid antihistaminics, Steroids, cardiac glycosides, which may worsen the situation.

- Local anesthetic proves better in the management of localized pain

- poly-valent anti venom may fail to produce the desired results

\section{CONCLUSION}

Domestically the scorpions are seen hiding in the gaps created by the cracking of tiles or cracking of the cement of the walls and they crawl out during night times avoiding light. One should pay attentions and the gaps should be either sealed off or cleaned regularly to decrease the harm hidden inside. Sometimes it is advised that the region of the sting must be tied with a tourniquet loosely to facilitate the hindrance to the absorption of the venom.

The lack of availability of scorpion venom anti-toxin is a point of major concern if it is available it is termed very costly, and it should be made available when ever required for the management of the scorpion sting cases at low rates.
A well-trained clinician must be made available in the emergency clinics for the management of the venomous sting cases as lack of management of the poisonous stings may lead to $30 \%$ of the fatalities.

\section{REFERENCES}

1. Tarachand S, Shailendar G, Maniram K. Scorpion bite causing acute severe myocarditis: A rare complication. Indian Journal of Clinical Practice. 2012:23(3);166-8.

2. Himmatrao SB, Pramodini HB. Scorpion Sting: Update. JAPI. 2012:60(1);46-55.

3. Ken D, Anthony PP, Norman AS. Scorpion Sting Syndrome - A Ten Year Experience. Annals of Saudi Medicine. 1995:15(2);148-55.

4. Hisham MAM. Scorpion sting syndrome: epidemiology, clinical presentation, and management of 2240 cases. Eastern Mediterranean Health Journal. 1997:3(1);82-9.

5. Satoskar RS, Bhandarkar SD, Nirmala NR. Immunotherapy, Immunosuppressants and Immunostimulants. Pharmacology and Pharmacotherapeutics. Revised $21^{\text {st }}$ edition, popular prakashan - Mumbai. 2009:1075-3

6. Prasad PS, Rudra JT, Vasanthi P, Sushitha U, Sadiq MJ, Narayana G. Assessment of drug use pattern using World Health Organization core drug use indicators at Secondary Care Referral Hospital of South India. CHRISMED J Health Res. 2015;2(3):223-8.

7. Himmatrao SB, Pramodini HB. Efficacy and safety of scorpion antivenom plus prazosin compared with prazosin alone for venomous scorpion (Mesobuthus tamulus) sting: randomised open label clinical trial. BMJ. 2011;342:c7136.

8. Chaturaka R, Ariaranee G. Management of scorpion envenoming: a systematic review and meta-analysis of controlled clinical trials. Rodrigo and Gnanathasan Systematic Reviews. 2017;6(74):1-12. 